\title{
A CASE OF PRIMARY ERYSIPELAS OF CONJUNCTIVA BULBI*
}

\author{
BY \\ N. I. Shimkin, M.D., Рh.D. \\ haifa, palestine
}

IT is probable that cases of primary erysipelas of the conjunctiva bulbi occur very seldom. I have not found any mention of such cases in books on infectious diseases, where much space is devoted to primary erysipelas of mucous membranes (Struempel, Kraus and Brugsch). In the widely used books on eye diseases (May, Morax, Fuchs, Axenfeld) much space is given to different secondary complications of the eyes during primary erysipelas of face and eyelids, but the possibility of primary erysipelas of the conjunctiva bulbi is never mentioned. Terrien and Coussin, who collected the literature on this question up to 1923, paid considerable attention to eye complications accompanying erysipelas of the face and of the eyelids, but they made no mention of the possibility of primary erysipelas on the conjunctiva bulbi. Only Groenouw, who collected the literature on erysipelas of the eye from 1857 to 1918 , mentions that "sometimes primary erysipelas starts from lachrymal ducts or from the conjunctiva" (p. 893). In the accessible periodical journals of recent years I have not found any information on this question.

Therefore I think it advisable to publish the first symptoms and the course of this rare disease which was investigated by me from beginning to the end. The diagnosis of the case was clear only 24 hours after the first examination of the patient, when per continuitatem erysipelas had passed on to the eyelids and face. The patient died unexpectedly on the fifth day of his illness from sudden cardiac failure.

History of the illness:-A man, aged 45 years. On 5th March, 1934, the patient was travelling in a train, when between 8 and 10 a.m. a foreign body came into his left eye. The patient rubbed his eye with a handkerchief, tears appeared and after a short time the symptoms subsided. The patient had been suffering continually from rheumatism and in his childhood had had several infectious diseases. In 1932 he had erysipelas faciei starting on the nose, and before that he had had erysipelas of the hand.

At about 11 p.m. on March 5, during a game of chess, he again complained of pain in his left eye, which grew worse every minute. Shortly afterwards pains on the left side of the head were added to those of the eye. These proved to be unbearable and they forced the patient to ring me up at 3.30 a.m. and ask for an immediate consultation which was fixed for 6 a.m.

March 6, 1934, 6 a.m. - The patient moaned and shivered on account of the severity of the pains. Spastic blepharospasm of the left eye. After one drop of 1 per cent.

*This paper was read at the meeting of the Jewish Medical Society in Haifa on March 20, 1934. 
pantocaine had been twice applied to the left eye, the patient opened the lids and stated that the pains in the eye and in the head had ceased. Examination of the left eye showed that the skin and the edges of the eyelids and also the naso-lacrymal ducts were normal. There was a slight hyperaemia of the palpebral conjunctiva, which is usually seen in pantocaine anaesthesia (Shimkin).

On the upper half of the conjunctiva sclerae, $1 \mathrm{~cm}$. above the corneal limbus, there were observed punctate haemorrhages resembling scratches on the conjunctiva due to some sharp and hard foreign body (when examined by Hartnack's magnifying glass). On the medial edge of the cornea in the region of the rima palpebrarum, was visible a limited oedema, such as is observed near the limbus at the time of a subconjunctival injection of 0.1 c.c. of any sterile and transparent liquid. The remaining surface of the conjunctiva bulbi was normal (see Fig. A). Tensionf $=16$ Schiötz). The pupil reactions and the fundus oculi were normal.

8 a.m. - Unbearable pains in the left eye and on the same side of the head, which only ceased for a short time after instilling 2 per cent. cocaine hydrochloride. On the conjunctiva sclerae instead of punctate haemorrhages there were three big ecchymoses measuring $2.5-4 \mathrm{mms}$. in diameter. The conjunctival oedema extended round $4 / 5$ of the surface of the cornea (see Fig. B). Tension, fundus oculi, normal; pupillary reactions normal. Pulse 68 ; temperature $36^{\circ} \mathrm{C}$.; lungs and heart normal.

4.30 p.m.-Patient had a rigor. Temperature $40^{\circ} \mathrm{C}$; ; heart, lungs, spleen, liver normal.

6 p.m.- Very severe pains in the left eye and in the head on the same side.

The conjunctiva round the cornea in the place of the former oedema was full of ecchymoses of dark blue colour protruded outside and nipped between the tightlysqueezed eyelíds (see Fig. C). In the conjunctival sac there was a minute quantity of fibrinous secretion which was taken for a microscopic examination. There was no detectable abnormality of the eyelids, of the mobility of the eyes, of the fundus oculi or of the tension.

March 7, 8 a.m.-Skin erysipelas of the external edges of the orbit and both lids of the left eye. The eyelids were swollen, red and tender when touched, and opened with difficulty. The pains in the left eye and in the left half of the head were greatly diminished. The spleen was enlarged. No further abnormality detected. Fibrinous secretion in the left conjunctival sac was more abundant than the day before. Smear was taken for microscopic examination.

March 8, 8 a.m.-Temperature $40^{\circ} \mathrm{C}$. Right eye : Erysipelas palpebrarum. Left eye : Erysipelas bullosum on the internal edge of the lower left eyelid. A transparent liquid was taken from the punctured bulla for microscopic examination The patient complained of the sight of his left eye continually becoming worse. The pains in the left eye and in the left half of the head were very insignificant. A pseudomembrane of fibrinous character was formed on the conjunctiva bulbi, which covered the cornea; this membrane was easily removed by a damp swab. This was taken for a microscopic examination. The cornea was slightly swollen, not transparent and therefore the fundus was not clear. The eyelids were opened by Desmarres' speculum. The mobility of the eyeball was slightly limited in all directions. Slight exophthalmos. Blood and urine were taken for pathological examination.

March 9, 8 a.m.-Temperature $39.8^{\circ} \mathrm{C}$. 7 p.m., temperature $40.3^{\circ} \mathrm{C}$. The pains in the left eye and in the left half of the head disappeared in the morning. The patient stated that his vision was much better than the day before. Exophthalmos and the limited mobility of the eyeball observed in the morning had almost disappeared towards the evening. Swelling, redness and tenderness of the lids of both eyes, greatly diminished in the morning and in the evening almost vanished from the right side. Erysipelas spread over the whole left side of the face, and the right and left sides of the neck. The glands of the neck were appreciably enlarged (erysipelas migrans).

The examination of the upper respiratory passages gave the following results (Dr. Gumpertz, oto-laryngologist):- "In the nose a moderate deviation of the septum to the left. The front part of the two inferior turbinate bones was missing, having been removed operatively from both sides, long ago. In the vestibulum nasi on both sides a slight formation of crusts. A slight atrophy of nasal mucous membrane, so that without making it anaemic one could examine from both sides the middle and upper nasal passages. No hint could be found as to secretion, which could be ascribed to the presence of any inflammation of the neighbouring sinuses. The laryngeal mucosa seemed slightly reddened. There were no signs of any stronger 
Erysipelas conjunctivae bulbi. ;ig. A

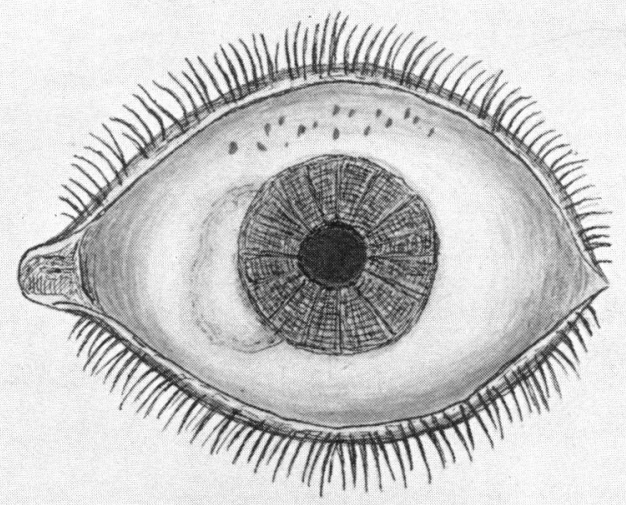

6.a.m
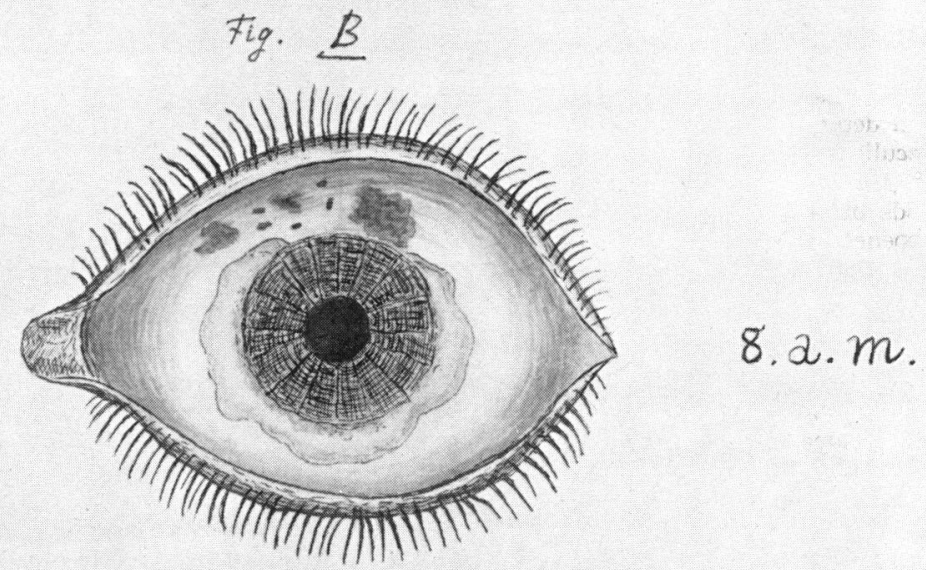

$$
\text { tiq. C }
$$

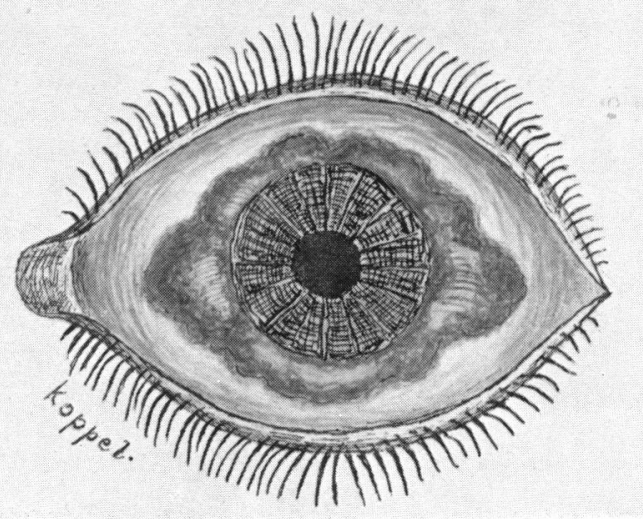

6.p.m. 
inflammation or oedema. The region of the frontal sinus and the front wall of the antrum sinus were not tender when pressed."

No abnormality whatever found in the heart. The spleen was appreciably enlarged.

From the bacteriological laboratory (Mrs. Dr. E. Shimkin) the following results of the investigations were given :- "Microscopic examination of the fibrinous secretion of the pseudo-membrane from the left conjunctiva of March 6, 7, 8 and 9 continually showed the presence of diplococcus lanceolatus capsulatus Gram-positive, of the type of pneumococcus Thalamon-Fraenkel. In the pseudo-membrane of March 9 together with diplococcus lanceolatus capsulatus were found some specimens of Grampositive short-chained streptococci."

In the liquid of punctured bullae some specimens of Gram-positive diplococci lanceolati capsulati of the type of pneumococcus Thalamon-Fraenkel were found.

Examination of Urine.-Albumin, 0.25 per cent. An acute positive reaction for urobilogen. The microscopic examination of sediment and all the other reactions were normal.

Blood Examination. - - Leucocyte, 4,400 ; polymorphonuclear (neutrophyl) 9 per cent. ; lymphocyte, 23 per cent. ; monocyte, 68 per cent.; haemoglobin, 65 per cent. ; erythrocyte, $3,550,000$; index, 0.9 .

March 10, 8 a.m.-Temperature $38.2^{\circ} \mathrm{C}$. The erysipelas of the left eyelids disappeared. The oedema of conjunctiva bulbi was almost invisible. The mobility of the eyeball was completely restored, the exophthalmos disappeared. The patient's vision was equally good in both eyes. The case had passed beyond the care of an ophthalmologist.

Dr. Nissenbaum (an internist, to whom I am obliged for the data of internal examination) took charge of the case. He stated that erysipelas covered completely the neck and a greater part of the chest. Sudden weakness of the heart was observed, and at 12 noon patient collapsed. At 2.25 p.m. patient passed away.

\section{Analysis of the Case}

From the history of the illness we see that in the left eye the scleral conjunctiva presented an oedema, haemorrhage and pains, preceding by 30 hours the appearance of erysipelas faciei and palpebrarum of the same side. Temperature and chill started 18 hours after unbearable pains in the left eye and in the left half of the head. The punctate haemorrhages that were observed at 6 a.m. became considerable ecchymoses after two hours. The limited pericorneal oedema of the conjunctiva bulbi occupied $4 / 5$ of the periphery of the cornea after two hours. 'Ten hours later an abundant ecchymosis was formed on the site previously occupied by oedema. The subjective symptom which brought the patient to the physician was the dull heavy pain in his left eye and the left half of his head, quickly diminishing after the application of 2 per cent. cocaine or 1 per cent. pantocaine, but starting again as soon as the action of the anaesthetic had ceased. The pain in the eye and the head disappeared in proportion to the spread of erysipelas on the eyelids and the face. At the first examination of the patient no disturbances of the skin of the

\footnotetext{
* N.B.-The leucopenia instead of leucocytosis in case of erysipelas, and monocytosis instead of usual polynucleosis was the result of previous blood diseases of the patient which preceded the erysipelas. Dr. Nissenbaum, internist, who examined the patient two months before his illness had found the same abnormal blood count. This abnormal blood condition was a signum mali, which weakened the defensive reaction of the body and hastened the death of the patient.
} 
eyelid or of its margins were observed. There were no signs of inflammation of nasal mucosa nor of any of the upper respiratory passages. During the whole time no pathological signs could be elicited from the lacrymal sac. The bacteriological examinations of fibrinous secretion and of pseudo-membrane of conjunctiva constantly showed the presence of Gram-positive diplococcus lanceolatus of the type of pneumococcus Thalamon-Fraenkel. The same diplococci were found in the liquid of punctured bullae. It must be also stated that the patient had had erysipelas twice previously and that a foreign body had come into his eye 13-14 hours before onset of pain, and that this foreign body had been washed out by the tears.

On the evidence of these symptoms and of the course of the disease, the case was diagnosed as one of primary erysipelas of the conjunctiva sclerae.

How can we explain the appearance of the subjective sensation of unbearable pain in the eye and the head, which seemed to contradict the insignificant findings of the conjunctiva sclerae? The medical history showed that the patient was prone to erysipelas as he had had it before on his hands and face. We also know that trauma was inflicted by a foreign body on the conjunctiva sclerae 13-14 hours before the appearance of pain in the eye and more than 30 hours before the onset of the general signs of erysipelas. It is known that the incubation period of erysipelas from the beginning of the infection till the appearance of the chill and of the temperature can be very short. The shortest period recorded has been seven to eight hours after infection (Kraus-Brugsch, p. 729). The sensation of pain and tension of the skin may precede the general painful phenomena of erysipelas (Struempel, p. 93). If erysipelas of skin and mucous membranes starts always from a sharply delimited and strongly reddened affected part, in this case it started on the scleral conjunctiva in a sharply delimited pericorneal oedema, disposed in the palpebral aperture; these peculiarities are explained by the special construction of the easily mobile tissue of the conjunctiva sclerae. Oedema and ecchymosis of the conjunctiva bulbi is a very common sign in any of the inflammatory processes of the superficial parts of the eye, besides as is known to every physician, oedema is always more clearly marked in the palpebral aperture, in consequence of the absence of the pressure of the eyelids in this region.

The minute trauma of conjunctival epithelium of the sclera by a foreign body opened the way to the infection. The punctate haemorrhages probably corresponded to the abrasions of conjunctiva. The unbearable pain in the left eye and in the left half of the head is a subjective symptom in a case of erysipelas, and it is caused, as has been explained above, by the tension and painfulness 
of the affected region of the tissue. The pain was made especially acute by pressing on this region. In this case there was the following vicious circle:- the painfulness and tension in the region of the affected conjunctiva evoked a spastic blepharospasm, and this latter gave rise to permanent pressure on the wounded part of the conjunctiva bulbi, causing even greater pain. This pain was transmitted reflexly to the left half of the head along the distribution of I and II branches of the trigeminal nerve, as the terminal ramifications of those branches are the sensory nerves of the conjunctiva of the eye. That is why in applying an anaesthetic to the sensory termination of the trigeminus nerve (a 2 per cent. solution of cocaine hydrochlor., or 1 per cent. solution of pantocaine) the pain in the left eye and in the left half of the head, as well as blepharospasm, disappeared immediately.

The quick onset and disappearance of oedema and of the high tension of different regions of tissue in cases of erysipelas migrans is a usual phenomenon; so it was in this case. When oedema and the tissue tension of the conjunctiva decreased on the third day of the illness, the pain in the eye and in the head simultaneously became less, and on the fourth day disappeared. The disappearance of the oedema was very clearly observed in this case, as was the serous infiltration of the tissues of the anterior part of the orbit. Owing to this serous infiltration there appeared on the third day of the illness limitation of the mobility of the eyeball and a slight exophthalmos, causing apprehension of the beginning of cellulitis of the orbit. All these symptoms disappeared on the fifth day of the illness. By this time the erysipelas had spread over the patient's neck and chest.

Independently from the above considerations, there arises the question whether in this case erysipelas could have been caused by diplococcus lanceolatus of the type of pneumococcus ThalamonFraenkel, as usually erysipelas is caused by streptococcus longus pathogenes s. erysipelatus.

Herrenschwand has collected a great deal of literature on the possibility of the passage of the diplococcus lanceolatus under certain conditions of culture into streptococcus haemolyticus and of the reverse process-streptococcus haemolyticus being transformed under certain conditions into viridans and then into typical pneumococcus.

Pfahler found in 66 out of 98 cases of erysipelas that the causative organism was a Gram-positive, non-mobile diplococcus, both intra- and extra-cellular; inoculated into a rabbit's ear it gave rise to a typical erysipelas.

Lehman and Neuman in their last edition of 1927 of "Bakteriologische Diagnostik" state that streptococcus lanceolatus, diplococcus pneumoniae Fraenkel and diplococcus lanceolatus 
sive lanceolatus capsulatus are synonyms of the same kind of bacterium, and besides this indicates that the pneumococcus may cause erysipelas (pp. 235-236).

In our case we invariably found diplococcus lanceolatus of the type of pneumococcus Thalamon-Fraenkel in all microscopic examinations of the fibrinous secretion and of the fibrinous pseudomembrane. And the character itself of fibrinous secretion and of pseudo-membrane correspond to that found in cases of pneumococcal conjunctivitis.

It is supposed that in the patient's conjunctival sac pathogenic pneumococci were present as parasitic organisms. They became virulent during the spring, when pneumococcal conjunctivitis frequently occurs (in Palestine about 2 per cent. of all conjunctivitis is due to pneumococci) (Shimkin).

It is particularly important that the same diplococcus of pneumococcus Thalamon-Fraenkel type was found in the contents of the bullae. The establishment of identity of bacteriological flora in the conjunctival secretion and in the bulla's fluid confirms once again the possibility of erysipelas being caused by pneumococcus of conjunctival origin. In the explanation of the epidemiology of primary erysipelas of the eye and eyelids this observation has a certain importance.

\section{Summary}

The author has described a case of primary erysipelas of the conjunctiva bulbi. The first symptoms, that brought the patient to the doctor, were the unbearable pains in the left eye and in the corresponding half of the head. A limited and not very extensive oedema of the conjunctiva in the region of the rima palpebrarum and punctate haemorrhages on the upper periphery of the conjunctiva sclerae quickly increased in size under observation.

The symptoms due to erysipelas of the eye were observed by the author 10 hours before the temperature and the chill started, and 24 hours before the appearance of erysipelas on the face, in consequence of the development per continuitatem of the infection from the conjunctiva bulbi on to the skin of the eyelids and on to the rest of the face.

The primary infection of erysipelas conjunctivae sclerae was, in all probability, caused by the pathological diplococcus sive streptococcus lanceolatus of the type of pneumococcus ThalamonFraenkel, that penetrated into the lymphatic vessels of the conjunc. tiva sclerae after its surface had been lacerated by a foreign body.

In examining a patient who complains of unbearable pain in the eye and in the corresponding side of the head, with such insignificant local signs in the conjunctiva bulbi a general practitioner 
should, in addition to excluding all the appropriate eye diseases, remember also the possibility of such a rare illness as primary erysipelas of conjunctiva bulbi, especially when the patient gives a history of previous attacks of erysipelas.

\title{
REFERENCES
}

1. Groenouw, A.-Graefe-Saemisch Handb. d. Augenheilk., III. Aufl., S. 893, 1920.

2. v. Herrenschwand, F.-Die pathogenen Mikroorganismen des Auges, S. 155. 1927.

3. Krauss, F, Brugsch, Th.-Spezielle Pathologie und Therapie innerer Krankheiten. II. Band : Infektionskrankheiten. II. Teil., 1919.

4. Lehman-Neumann -Bakteriologische Diagnostik. 2 Aufl., II. Band., SS. 235-236, 1927.

5. Panton and Adams.-An investigation in the aetiology of the Erysipelas allied infection. Lancet, Vol. II, p. 1065, 1909.

6. Pfahler.-The Bacteriology of Erysipelas. Philad. Med. Jl., April 19, 1902.

7. Struempel, Ad.-Lehrbuch der speziellen Pathologie und Therapie der inneren Krankheiten. Bd. I., XXII. Aufl., 1920.

8. Shimkin, N.-Pantocain, a new anaesthetic in eye practice. Folia ophthalmologica orientalia. Fasc. 2, p. 195, 1933.

Blindness among Jews in Palestine and its causes. Congrès International de Médecine tropical et d'Hygiène. Le Caire, Egypt, Décembre, 1928. Compte Rendu, Vol. III, p. 837, 1931. Le Caire.

9. Terrien, F., and G. Coussin.-Affection de l'oeil en médecin général. 1924.

\section{THE X-RAY TREATMENT OF BLEPHARITIS*}

BY

\author{
Nessim Abu-Saif, M.R.C.S.(Eng.), D.M.R.E.(Camb.) \\ ASSISTANT RADIOLOGIST, KASR-EL-AINI HOSPITAL, CAIRO, LECTURER \\ IN RADIOLOGY, FACULTY OF MEDICINE, CAIRO
}

\section{Pathology}

Two main varieties of blepharitis are known, viz.-

1. Blepharitis squamosa.-Here the lid margin is reddened, the palpebral conjunctiva is hyperaemic and the space between the cilia is filled with small thin whitish scales resembling dandruff. The scales can be removed imperfectly by washing or more thoroughly by forceps, leaving the lid margin reddened and succulent, but not ulcerated. The cilia fall out easily. They soon grow again.

Instead of the scaly formation mentioned, the lid margin may present yellow crusts with no underlying ulceration, due to the drying of the excessive sebaceous secretion.

* Read before the Egyptian Medical Association and the Ophthalmological Society of Egypt at its Annual General Meeting in March, 1934. 